\title{
Thermal Degradation of Single-Walled Carbon Nanotubes
}

Hai M. Duong, Erik Einarsson, Jun Okawa, Rong Xiang, Shigeo Maruyama*

Department of Mechanical Engineering, The University of Tokyo

7-3-1 Hongo, Bunkyo-ku, Tokyo 113-8656, Japan

*E-mail address: maruyama@photon.t.u-tokyo.ac.jp

\begin{abstract}
An optical absorbance technique was used to study the burning temperature and burning mechanism of vertically aligned single-walled carbon nanotube (VA-SWNT) films. Use of this simple optical method is shown to be consistent with the standard thermogravimetric analysis (TGA) method, but can be applied to a very small amount of SWNTs. Experimental results indicate burning of the VA-SWNTs is not localized, but occurs throughout the film. Furthermore, thicker films have a slightly higher burning temperature than thinner films synthesized under the same conditions. This is believed to be due to a higher bundle density and more uniform distribution of SWNTs within the film.
\end{abstract}

KEYWORDS: SWNT, Thermal Degradation, Density, Optical absorption, TGA 


\section{Introduction}

Due to their many unique properties, single-walled carbon nanotubes (SWNTs) are promising for use in composite materials to enhance mechanical properties as well as electrical $^{1)}$ and thermal ${ }^{2,3)}$ transport. A major challenge currently facing practical device development is how to control the morphology of SWNTs. The combination of a dip-coat catalyst loading process ${ }^{4}$ ) for deposition of $\mathrm{Co} / \mathrm{Mo}$ bi-metal catalyst particles with the alcohol catalytic CVD method $^{5)}$ has shown to produce uniform films of vertically aligned SWNTs (VA-SWNTs) over square-centimeter areas. ${ }^{6}$ The density of the VA-SWNT film has previously been estimated ${ }^{7)}$ to be between 0.035 and $0.06 \mathrm{~g} / \mathrm{cm}^{3}$, but has not yet been accurately determined.

The standard way to determine the burning temperature of a material is by thermal gravimetric analysis (TGA), where one measures the change in mass of a material as it is heated in a well-controlled manner. The problem with applying TGA to VA-SWNTs is that it is often difficult to obtain sufficient mass for an accurate measurement. In this study, we introduce a method for measuring the burning temperature of VA-SWNT films and investigating the burning mechanism using an optical absorbance measurement. By this technique, which we call thermal optical absorbance (TOA), burning of the film is determined by measuring the change in intensity of an incident laser during heating of the film. This is essentially a modification of an in situ optical method previously used to study the growth process of VA-SWNTs, ${ }^{8,9)}$ and is shown to be accurate using a small amount of material. From these measurements, we determine the density of the VA-SWNT films and compare these results to measurements performed using the standard TGA technique. The TOA method is also used to investigate the thermal degradation of VA-SWNT films with different thicknesses.

\section{Experimental Methods}

\subsection{VA-SWNT synthesis}

VA-SWNTs were synthesized on quartz substrates using the alcohol catalytic CVD process. $^{5,6)}$ The catalyst was supported on a quartz substrate by dip-coating in a solution of Co and Mo acetates dissolved in ethanol (metal content $0.01 \mathrm{wt} \%$ each). The catalyst was oxidized by heating the dip-coated substrate in air at $400{ }^{\circ} \mathrm{C}$, and then reduced in a flowing $\mathrm{Ar} / \mathrm{H}_{2}$ mixture $\left(3 \% \mathrm{H}_{2}, 300 \mathrm{sccm}\right.$ flow rate) during heating of the CVD chamber. The resulting catalyst particles have diameters of 1-2 nm, from which SWNTs with similar diameters are synthesized. ${ }^{10,11)}$ After the CVD chamber had reached $800{ }^{\circ} \mathrm{C}$, the $\mathrm{Ar} / \mathrm{H}_{2}$ flow was stopped, and SWNT films were synthesized by supplying ethanol vapor (99.5\% dehydrated ethanol) at typically $1.4 \mathrm{kPa}$ (10 Torr) for a few minutes, depending on the desired film thickness. Figure 1a shows the thickness of a VA-SWNT film throughout the CVD reaction time. A scanning electron microscopy (SEM) image of the 
VA-SWNT film (Fig. 1b) shows the thickness is approximately $28 \mu \mathrm{m}$.

The film thickness was monitored during growth using an in situ absorbance measurement, where a 488-nm $\mathrm{Ar}^{+}$laser was guided into the CVD chamber through an aperture (4 $\mathrm{mm}$ diameter) in the wall of the annular electric furnace. The quartz substrate was positioned in the chamber such that the laser light is incident normal to the substrate surface. The transmitted laser light leaves the chamber through an aperture on the opposite side of the furnace, and its intensity is measured and recorded using a computer-based data-logging system. The attenuation in the laser intensity is used to calculate the absorbance of the sample throughout the CVD reaction. The growth curve plots the film thickness (which is proportional to the absorbance) throughout CVD.

\subsection{Thermal measurements}

Standard TGA experiments were performed using a TGA 6300 (Seiko Instruments Inc.), which has an accuracy of $0.001 \mathrm{mg}$. VA-SWNT samples of different thickness were removed from their quartz substrates by immersion into $60{ }^{\circ} \mathrm{C}$ water, ${ }^{12)}$ and then collected and measured. The SWNTs were initially heated from room temperature to $150{ }^{\circ} \mathrm{C}$ at a heating rate of $2{ }^{\circ} \mathrm{C} / \mathrm{min}$, and then held at that temperature for 4 hours to remove any water contained in the sample. The SWNTs were then heated to $1000{ }^{\circ} \mathrm{C}$ at $2{ }^{\circ} \mathrm{C} / \mathrm{min}$.

The burning temperatures of various VA-SWNT films were also determined by the optical TOA method, where the aforementioned in situ absorbance measurement was performed during well-controlled heating of the film. VA-SWNTs were heated from room temperature to $100{ }^{\circ} \mathrm{C}$ in 10 minutes, held at $100{ }^{\circ} \mathrm{C}$ for 30 minutes to desorb any water, and then heated to $800-1000{ }^{\circ} \mathrm{C}$ at a rate of $2{ }^{\circ} \mathrm{C} / \mathrm{min}$. The heating environment was dry air at atmospheric pressure. Burning of the VA-SWNT films is detected from the resulting decrease in absorbance. ${ }^{9)}$ Unlike the whole-sample measurement of standard TGA, the TOA technique can only measure burning over a small area of the VA-SWNT film. However, this small area is sufficient to obtain an accurate measurement.

\section{Results and Discussion}

Here we discuss the morphology, density, and burning of VA-SWNT films synthesized at different ethanol pressures. The films were characterized using resonance Raman and optical absorbance spectroscopy. Thermal degradation of the VA-SWNT films is compared using two different methods.

\subsection{Growth and structure of the SWNT films}

VA-SWNTs were synthesized at $0.8,1.5$, and $3.0 \mathrm{kPa}$, and were grown until the film thickness had reached approx. $5 \mu \mathrm{m}$. The film thickness was monitored during growth by the in situ absorbance measurement described in Section 2.1. The corresponding 
growth curves of the VA-SWNT films are shown in Fig. 2a. Resonance Raman scattering spectra (Fig. 2b) taken from the 5- $\mu \mathrm{m}$ films indicate the SWNTs are of good quality, as evidenced by the small D-band near $1350 \mathrm{~cm}^{-1}$ relative to the dominant G-band. The radial breathing mode (RBM) peaks below $300 \mathrm{~cm}^{-1}$ are characteristic of VA-SWNTs measured from the top of the sample and excited using a 488-nm laser. ${ }^{13)}$

Optical absorbance of the VA-SWNT films (Fig. 3) was measured using a UV-vis-NIR spectrophotometer equipped with a rotational substrate holder and a polarizer. ${ }^{14)}$ Absorbance spectra corresponding to incidence angles of $0,15,30$, and $45^{\circ}$ are shown in Fig. 3a. At $0^{\circ}$, the incident light is polarized perpendicular to the SWNT orientation axis, and the electric field vector becomes more parallel to the SWNTs as the incident angle increases. This results in increased absorbance by both the parallel-excitation component of strong $\pi$-plasmon ${ }^{14)}$ near $4.5 \mathrm{eV}$ and the interband transitions near $1 \mathrm{eV}$. Since the interband absorption energies are related to the SWNT diameter, the positions of these peaks reflect the diameter distribution of the SWNTs in the aligned film. These peaks are shown in detail for normal $\left(0^{\circ}\right)$ incidence in Figure $3 \mathrm{~b}$. There is very little change in the spectra, except for a slight shift in peak positions for VA-SWNTs synthesized at $3.0 \mathrm{kPa}$, indicating a slightly smaller diameter distribution.

\subsection{Determination of VA-SWNT density}

Three different samples, consisting of a quartz substrate and VA-SWNTs, were carefully weighed prior to burning. The samples were then heated in air to $1000{ }^{\circ} \mathrm{C}$ to ensure complete burning of the SWNTs. After burning, the bare substrate was again carefully weighed, and the mass of the burned SWNTs determined from the mass difference. Values obtained by this method were between 0.57 and $0.94 \mathrm{mg}$. The volumes of the burned VA-SWNT films were determined from the area of the burned sample and the film thickness, which had been pre-determined by SEM observation. The VA-SWNT film thicknesses were from 17.5 to $25 \mu \mathrm{m}$, and the sample areas ranged from 237.5 to $360.5 \mathrm{~mm}^{2}$. The overall densities obtained for the VA-SWNT films are shown in Table I. Although it was found that thicker films have a slightly higher density, the average density was found to be $0.05 \pm 0.005 \mathrm{~g} / \mathrm{cm}^{3}$.

\subsection{Thermal degradation of VA-SWNT films}

Figure 4 shows the burning temperature of an 11- $\mu \mathrm{m}$-thick SWNT film, determined using the optical absorbance technique, and of a 10- $\mu$ m-thick SWNT film, determined using the standard TGA method. Both methods yield burning temperatures of approx. $525{ }^{\circ} \mathrm{C}$. This shows the TOA method, which is better suited for small amounts of SWNTs, is consistent with TGA results, thus can be reliably applied to study the thermal degradation of SWNTs.

VA-SWNT films originally having a thickness of $5 \mu \mathrm{m}$ were burned by approximately $30,60,75,85$ and $90 \%$, as determined from the absorbance measurement. 
Absorbance spectra obtained before and after different degrees of controlled burning are shown in Fig. 5. The morphology change due to this burning was investigated by SEM observation. Cross-sectional SEM images of the VA-SWNT films obtained after different stages of burning are shown Figs. 6a-e. Although the absorbance spectra in Fig. 5 show the film was burned by $60 \%$ in the first two steps, the film thickness only decreases by $35 \%$. This means the film was not burned primarily at the top, but throughout the entire film. This may be due to defective and/or isolated SWNTs burning first. As burning continues the film thickness decreases still does not match the extent of burning indicated by optical absorbance (Fig. 5), indicating a further decrease in SWNT density. Severe burning of the films (Figs. 7a-7c) results in a very low SWNT density, which eventually leads to a collapse of the vertically-aligned structure. At 90\%, shown in Fig. 7c, the SWNTs are no longer aligned, and some areas appear completely burned. This collapse of the film, however, causes the relative polarization of the incident light to change. Hence, the optical absorbance should over-estimate the mass of SWNTs. Because of the non-uniformity of collapse, quantitative analysis of this effect is challenging.

To check the influence of high temperature exposure without burning, 8 - $\mu \mathrm{m}$-thick VA-SWNT films were heated in both dry air and $\mathrm{Ar} / \mathrm{H}_{2}$ atmospheres (Fig. 8a). In dry air, the SWNT film starts burning at approx. $400{ }^{\circ} \mathrm{C}$, and is completely burned by $600{ }^{\circ} \mathrm{C}$. In the $\mathrm{Ar} / \mathrm{H}_{2}$ environment, the VA-SWNTs withstand temperatures as high as $1000{ }^{\circ} \mathrm{C}$. Figure $8 \mathrm{~b}$ shows resonance Raman spectra (488-nm excitation) of the films before and after heating in the $\mathrm{Ar} / \mathrm{H}_{2}$ environment. The two spectra are nearly identical, indicating the structure of the VA-SWNT film was unchanged by exposure to a high temperature environment in which no burning occurred.

Figure 9a shows burning curves obtained by the standard TGA measurement for two VA-SWNT films with thicknesses of 10 and $17 \mu \mathrm{m}$. The burning temperatures of these two films (Fig. 9b) are found to be 525 and $545{ }^{\circ} \mathrm{C}$, respectively. The slope of the TGA curves in the low-temperature region is due to drift in the machine (purple baseline in Fig. 9a), and is not a feature of the sample. The thickness-dependence on thermal degradation may be explained by the higher density of the thicker VA-SWNT films (see Table I), which should have more bundles and fewer isolated SWNTs within the film. It is also possible that thicker VA-SWNT films (e.g. $>20 \mu \mathrm{m}$ ) contain a fraction of double-walled and/or multi-walled nanotubes, which would be more stable, thus have a higher burning temperature.

The advantage of the optical absorbance technique is to use a very small amount of SWNTs to determine their burning temperature. Figure 10 shows the burning curves of three different SWNTs with thicknesses of 8, 11 and $29 \mu \mathrm{m}$. This figure shows that thicker SWNT films have higher burning temperatures, in agreement with the TGA 
results (Fig. 9). The data obtained by the standard TGA method, seen in Figs. 9b and 10, indicates that burning occurs over a broader temperature range than for the TOA method. This may be due to more precise and feed-back controlled temperature in the TGA system, but further study is necessary to clarify this inconsistency. Because of the simple control of ambient temperature in our TOA method, applicability to heavily exothermic reaction is limited.

\section{Conclusions}

We have investigated the thermal degradation of VA-SWNT films using an optical absorbance technique, which is shown to be well-suited for low-mass materials such as VA-SWNTs. Combined with SEM observation and absorbance spectroscopy, we find that thermal degradation of VA-SWNTs occurs throughout the film, rather than in a specific location, e.g. at the top of the film. Severe burning causes the film density to decrease to the point of collapse of the aligned structure. Both optical (TOA) and TGA results show that thicker VA-SWNT films synthesized under the same conditions delay the thermal degradation process. This is believed to be due to denser packing and more uniform distribution of SWNTs within the vertically aligned film. Possible formation of fraction of double-walled nanotubes in the thicker films, however, is another possible explanation for the higher thermal stability. 


\section{REFERENCES}

1) P.M. Ajayan, L.S. Schadler, C. Giannaris, A. Rubio: Adv. Mater. 12 (2000) 750.

2) S.U.S. Choi, Z.G. Zhang, W. Yu, F.E. Lockwood, E.A. Grulke: Appl. Phys. Lett. 79 (2001) 2252.

3) M.J. Biercuk, M.C. Llaguno, M. Radosavljevic, J.K. Hyun, A.T. Johnson, J.E. Fischer: Appl. Phys. Lett. 80 (2002) 2767.

4) Y. Murakami, Y. Miyauchi, S. Chiashi, S. Maruyama: Chem. Phys. Lett. 377 (2003) 49.

5) S. Maruyama, R. Kojima, Y. Miyauchi, S. Chiashi, M. Kohno: Chem. Phys. Lett. 360 (2002) 229.

6) Y. Murakami, S. Chiashi, Y. Miyauchi, M. Hu, M. Ogura, T. Okubo, S. Maruyama: Chem. Phys. Lett. 385 (2004) 298.

7) Y. Murakami: PhD Thesis, Department of Mechanical Engineering, The University of Tokyo, Dec 2004, p.128.

8) S. Maruyama, E. Einarsson, Y. Murakami, T. Edamura: Chem. Phys. Lett. 403 (2005) 320.

9) E. Einarsson, Y. Murakami, M. Kadowaki, S. Maruyama: Carbon, submitted.

10) M. Hu, Y. Murakami, M. Ogura, S. Maruyama, T. Okubo: J. Catal. 225 (2004) 230.

11) Y. Murakami, E. Einarsson, T. Edamura, S. Maruyama: Carbon 43 (2005) 2664.

12) Y. Murakami and S. Maruyama: Chem. Phys. Lett. 422 (2006) 575.

13) Y. Murakami, S. Chiashi, E. Einarsson, S. Maruyama: Phys. Rev. B 71 (2005) 085403.

14) Y. Murakami, E. Einarsson, T. Edamura, S. Maruyama: Phys. Rev. Lett. 94 (2005) 087402. 


\section{FIGURE CAPTIONS}

Fig. 1. (a) VA-SWNT film thickness during CVD growth measured by the in situ absorbance method. (b) Cross-sectional SEM image of the resulting VA-SWNT film.

Fig. 2. (a) Growth curves of VA-SWNT films synthesized at different pressures. The growth was stopped upon reaching $5 \mu \mathrm{m}$ to produce films with similar thickness. (b) Resonance Raman spectra $(\lambda=488 \mathrm{~nm})$ of the films, with RBM peaks in the inset.

Fig. 3. (a) VA-SWNT film absorbance of p-polarized light at incident angles of 0, 15, 30, and $45^{\circ}$. (b) Low-energy interband absorption peaks for normal incidence $\left(0^{\circ}\right)$. A slight shift is seen for VA-SWNTs synthesized at higher ethanol pressure $(3.0 \mathrm{kPa})$, indicating a smaller diameter distribution.

Fig. 4. Comparison of TGA and TOA methods for measuring thermal degradation of VA-SWNT films having nearly the same thickness and synthesized under the same conditions.

Fig. 5. Optical absorbance spectra of VA-SWNT films before burning (dotted line) and after burning by $30,60,75,85$, and $90 \%$ (listed in order from upper to lower curve).

Fig. 6. Cross-sectional SEM images of VA-SWNT films (a) before burning and after burning by (b) $30 \%$, (c) $60 \%$, (d) $75 \%$, and (e) $85 \%$. Note the change in film thickness does not match the extent of burning, indicating burning occurs throughout the film rather than at the top of the film. Scale-bar in (a) applies to all images.

Fig. 7. SEM images of the VA-SWNT films burned by (a) $75 \%$, (b) $85 \%$ and (c) $90 \%$. Collapse of the aligned structure is apparent in (b) and (c). Scale-bar in (a) applies to all images.

Fig. 8. (a) TOA measurements of 8- $\mu$ m-thick VA-SWNT films heated in 1 atm of dry air (blue curve) and $\mathrm{Ar} / \mathrm{H}_{2}$ (red curve). No burning is observed when heated in $\mathrm{Ar} / \mathrm{H}_{2}$, but heating in dry air shows burning occurs at $\sim 500^{\circ} \mathrm{C}$ (see inset). (b) Resonance Raman spectra, with RBM inset, of the 8- $\mu$ m-thick VA-SWNT film before (thick black line) and after (thin red line) heating in $\mathrm{Ar} / \mathrm{H}_{2}$. No change is apparent after heating.

Fig. 9. (a) Thermal degradation obtained using the standard TGA measurement of VA-SWNT films synthesized under the same experimental conditions. Purple line is 
baseline due to machine drift. (b) The peaks in the DTG spectra show the burning temperature of the two films.

Fig. 10. Thermal degradation measurement obtained using the TOA method for VA-SWNT films synthesized under the same conditions but grown to different thickness. The thicker films show higher thermal stability. 


\section{TABLES}

Table I. Density of VA-SWNT films of different thicknesses

\begin{tabular}{|c|c|}
\hline VA-SWNT film thickness $[\mu \mathrm{m}]$ & VA-SWNT film density $\left[\mathrm{g} / \mathrm{cm}^{3}\right]$ \\
\hline approx. 10 & 0.035 \\
approx. 17 & 0.045 \\
approx. 25 & 0.055 \\
\hline
\end{tabular}




\section{FIGURES}
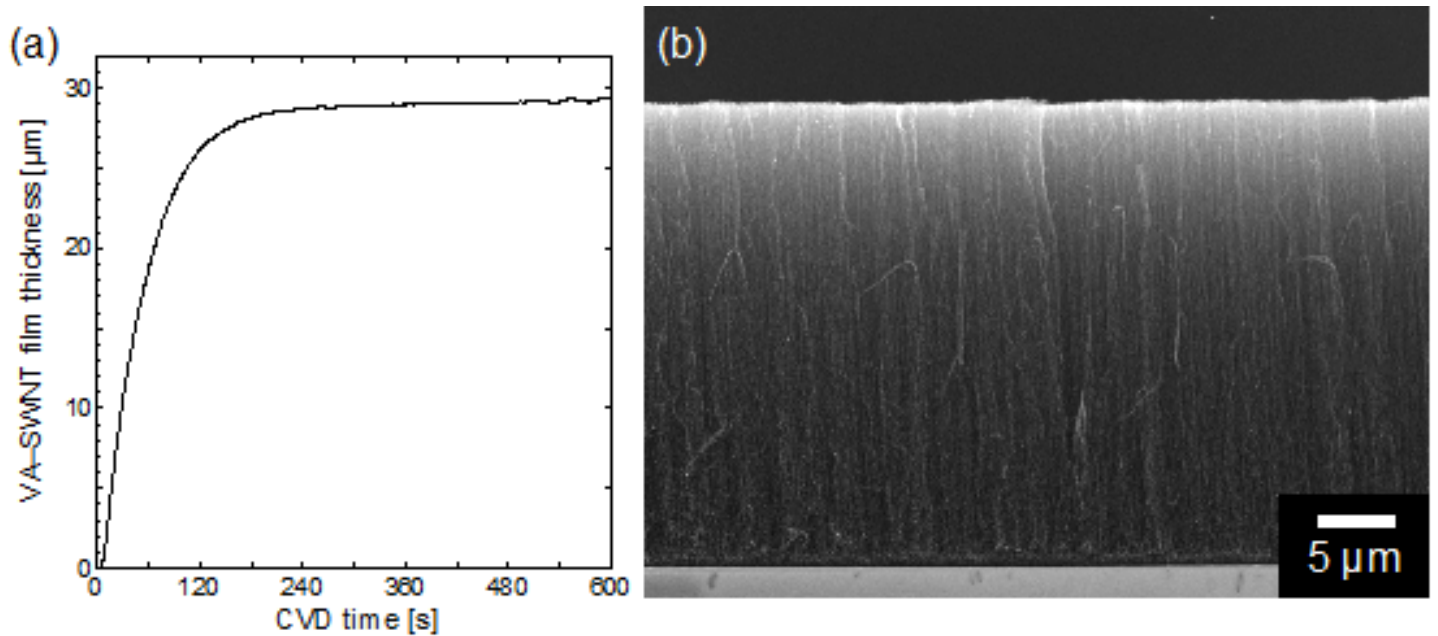

Fig. 1. (a) VA-SWNT film thickness during CVD growth measured by the in situ absorbance method. (b) Cross-sectional SEM image of the resulting VA-SWNT film.
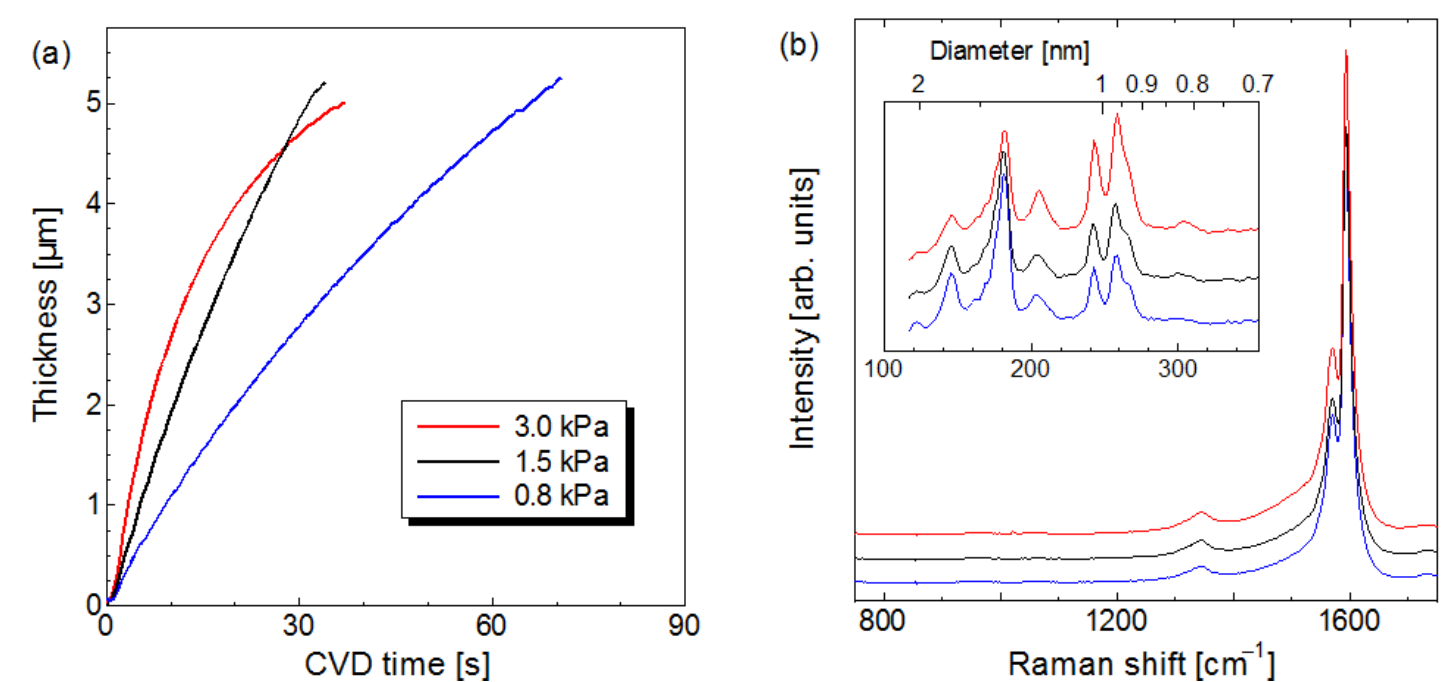

Fig. 2. (a) Growth curves of VA-SWNT films synthesized at different pressures. The growth was stopped upon reaching $5 \mu \mathrm{m}$ to produce films with similar thickness. (b) Resonance Raman spectra $(\lambda=488 \mathrm{~nm})$ of the films, with RBM peaks in the inset. 

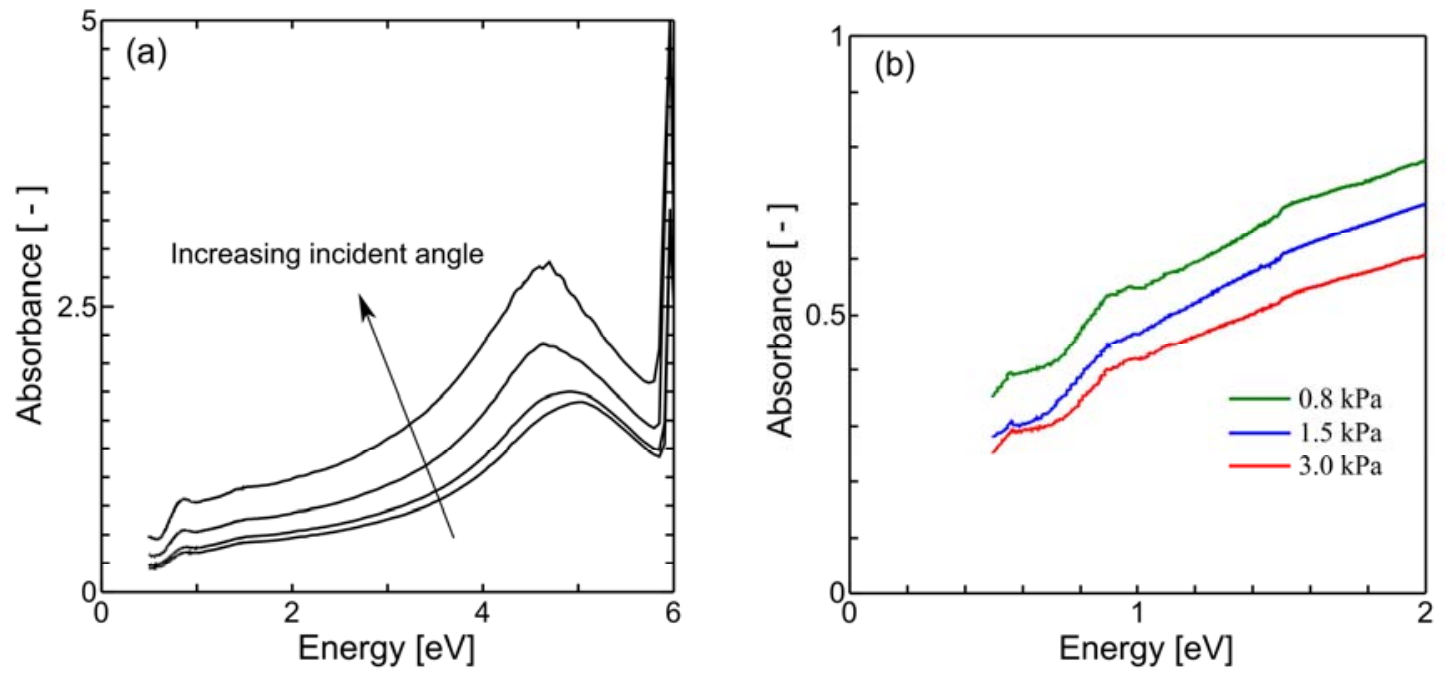

Fig. 3. (a) VA-SWNT film absorbance of p-polarized light at incident angles of 0, 15, 30, and $45^{\circ}$. (b) Low-energy inter-band absorption peaks for normal incidence $\left(0^{\circ}\right)$. A slight shift is seen for VA-SWNTs synthesized at higher ethanol pressure $(3.0 \mathrm{kPa})$, indicating a smaller diameter distribution.

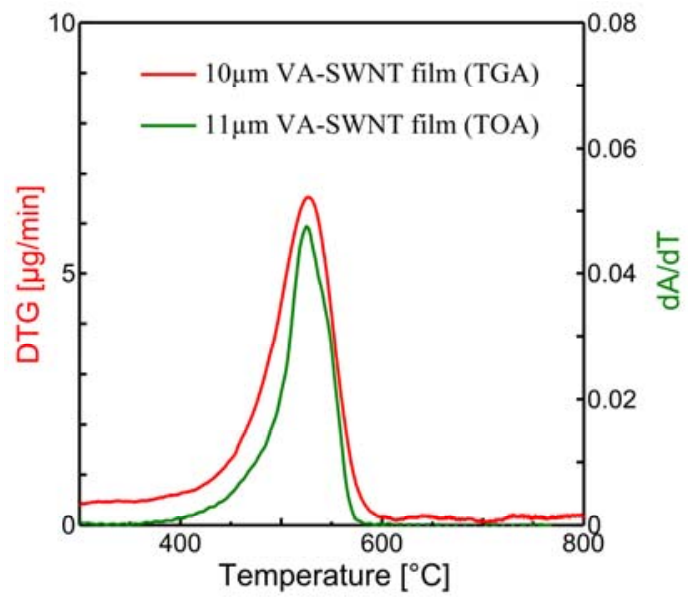

Fig. 4. Comparison of TGA and TOA methods for measuring thermal degradation of VA-SWNT films having nearly the same thickness and synthesized under the same conditions. 


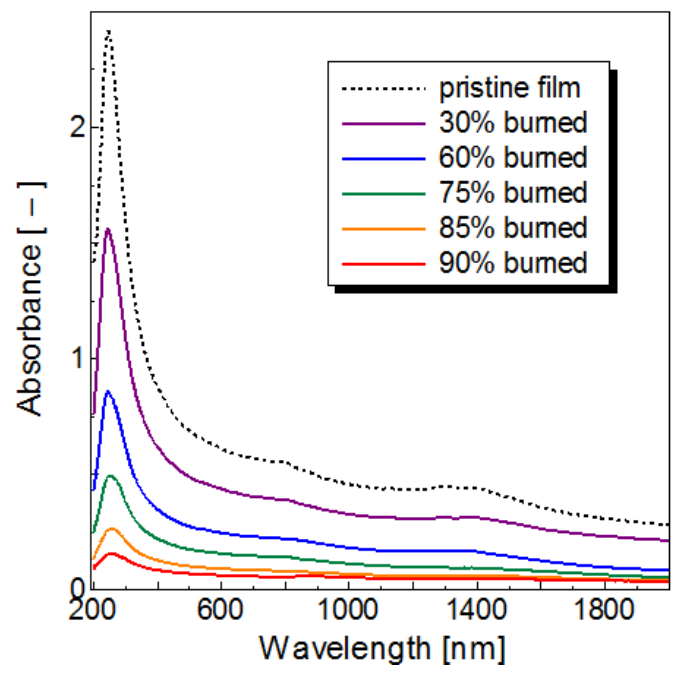

Fig. 5. Optical absorbance spectra of VA-SWNT films before burning (dotted line) and after burning by $30,60,75,85$, and $90 \%$ (listed in order from upper to lower curve).

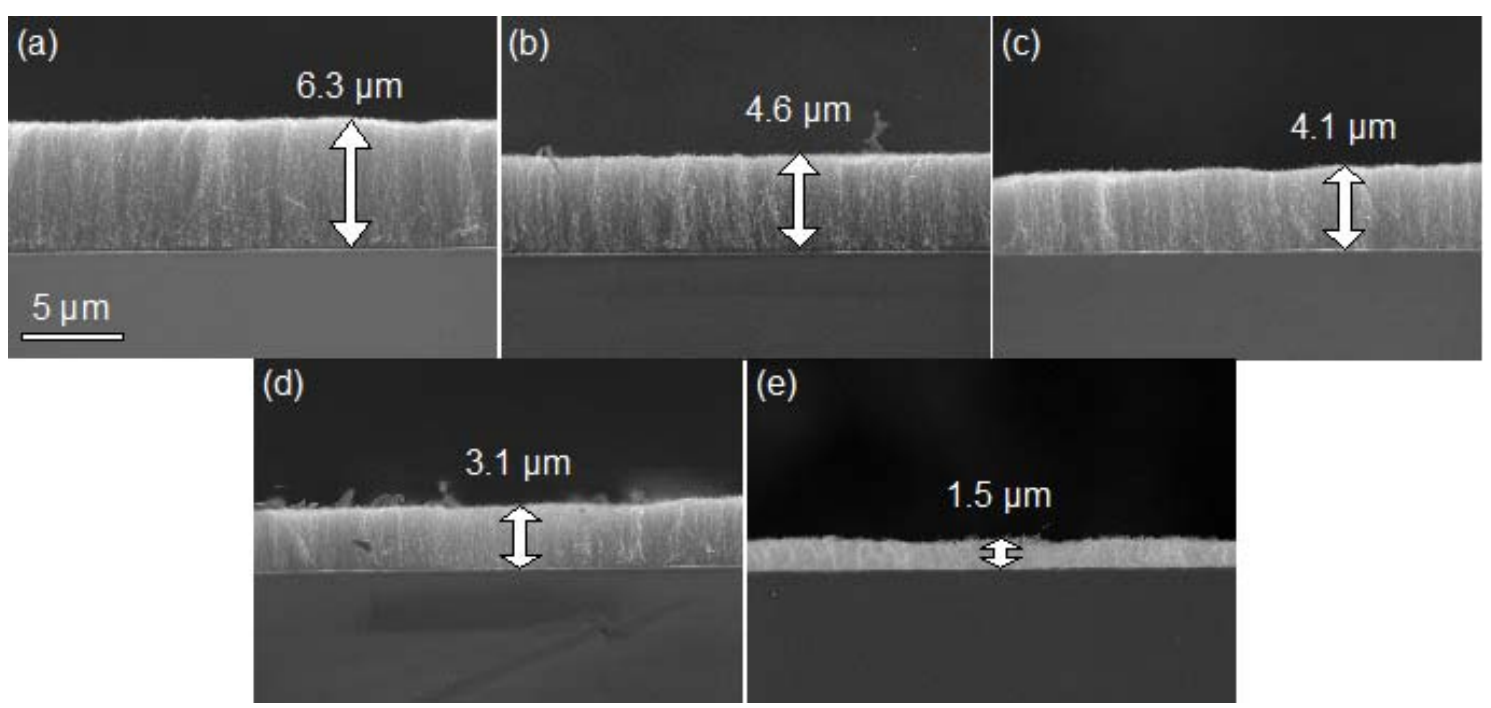

Fig. 6. Cross-sectional SEM images of VA-SWNT films (a) before burning and after burning by (b) $30 \%$, (c) $60 \%$, (d) $75 \%$, and (e) $85 \%$. Note the change in film thickness does not match the extent of burning, indicating burning occurs throughout the film rather than at the top of the film. Scalebar in (a) applies to all images. 

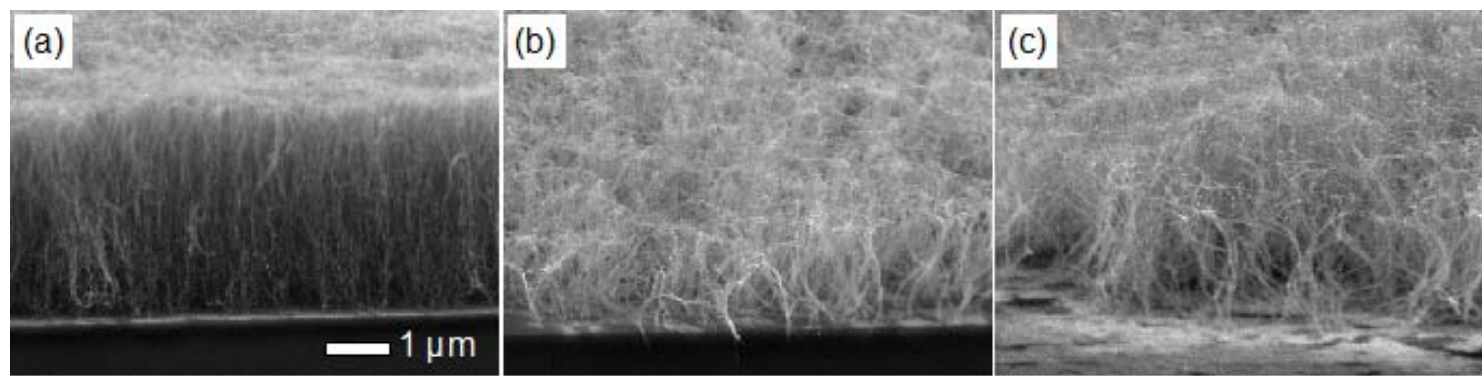

Fig. 7. SEM images of the VA-SWNT films burned by (a) $75 \%$, (b) $85 \%$ and (c) $90 \%$. Collapse of the aligned structure is apparent in (b) and (c). Scalebar in (a) applies to all images.
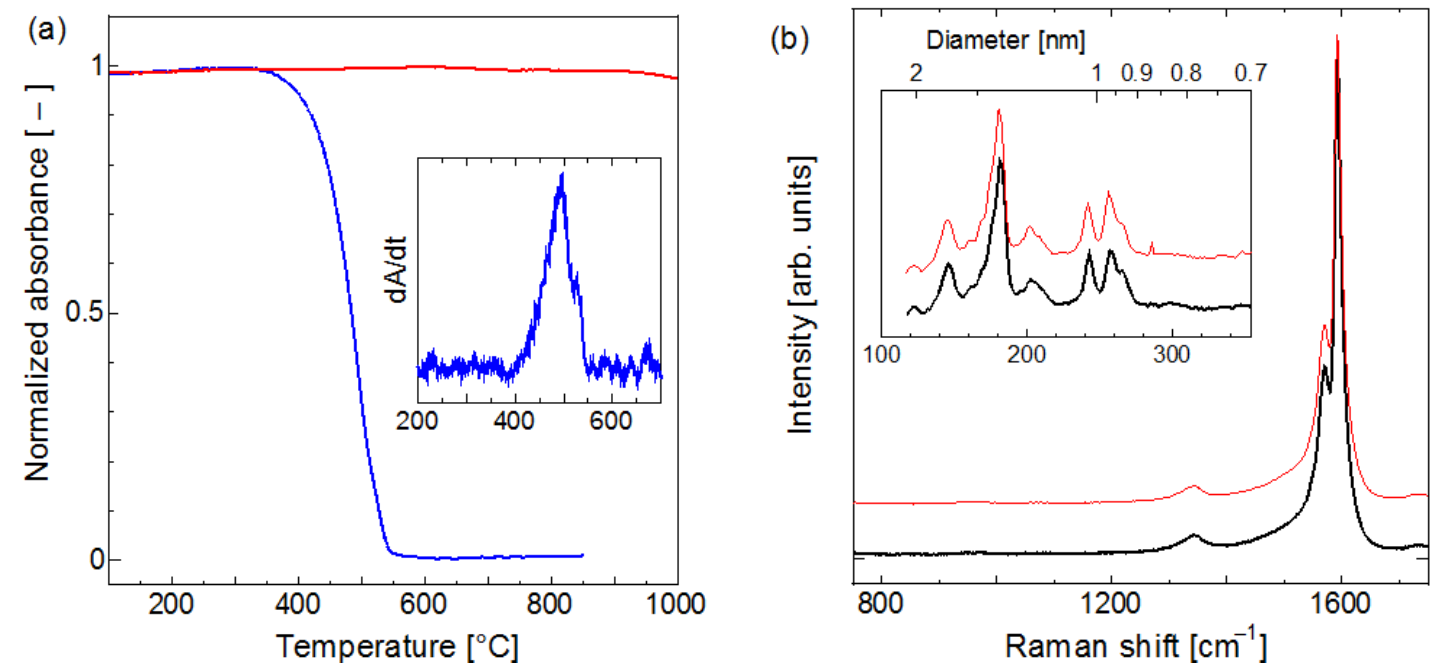

Fig. 8. (a) TOA measurements of 8- $\mu$ m-thick VA-SWNT films heated in 1 atm of dry air (blue curve) and $\mathrm{Ar} / \mathrm{H}_{2}$ (red curve). No burning is observed when heated in $\mathrm{Ar} / \mathrm{H}_{2}$, but heating in dry air shows burning occurs at $\sim 500{ }^{\circ} \mathrm{C}$ (see inset). (b) Resonance Raman spectra, with RBM inset, of the 8- $\mu$ m-thick VA-SWNT film before (thick black line) and after (thin red line) heating in $\mathrm{Ar} / \mathrm{H}_{2}$. No change is apparent after heating. 

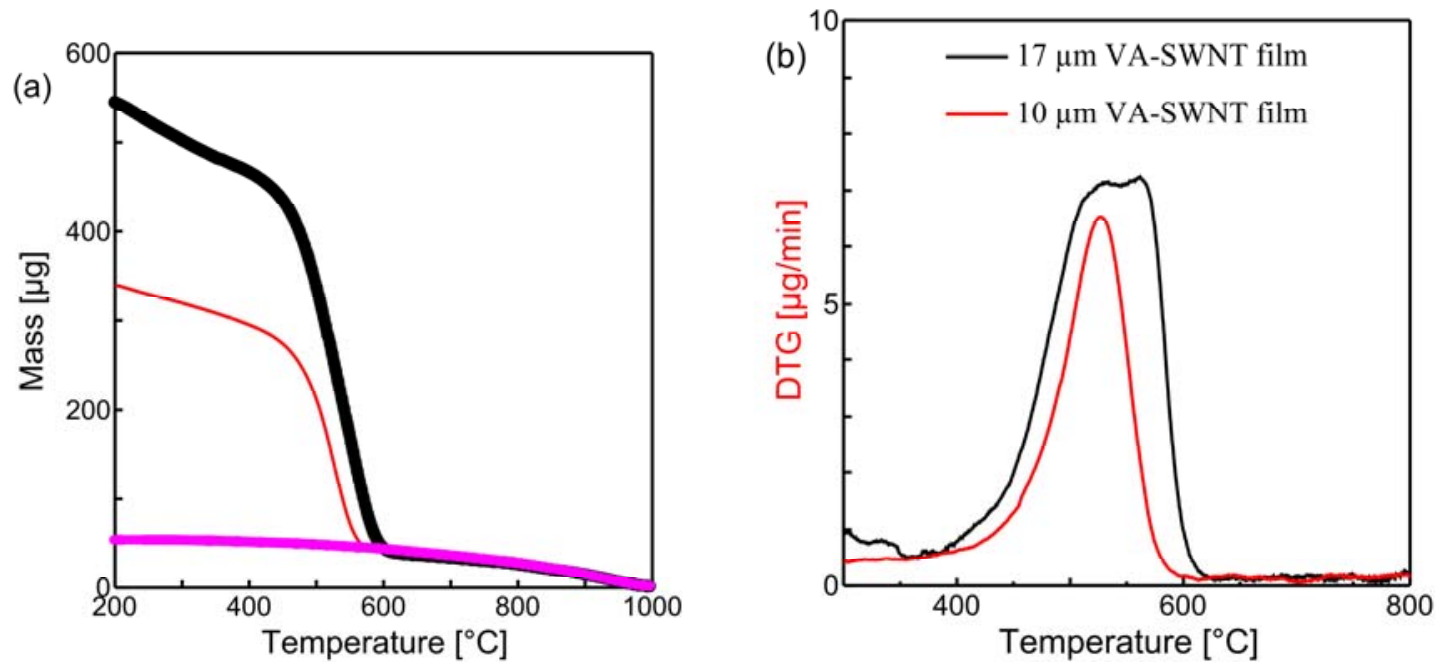

Fig. 9. (a) Thermal degradation obtained using the standard TGA measurement of VA-SWNT films synthesized under the same experimental conditions. Purple line is baseline due to machine drift. (b) The peaks in the DTG spectra show the burning temperature of the two films.

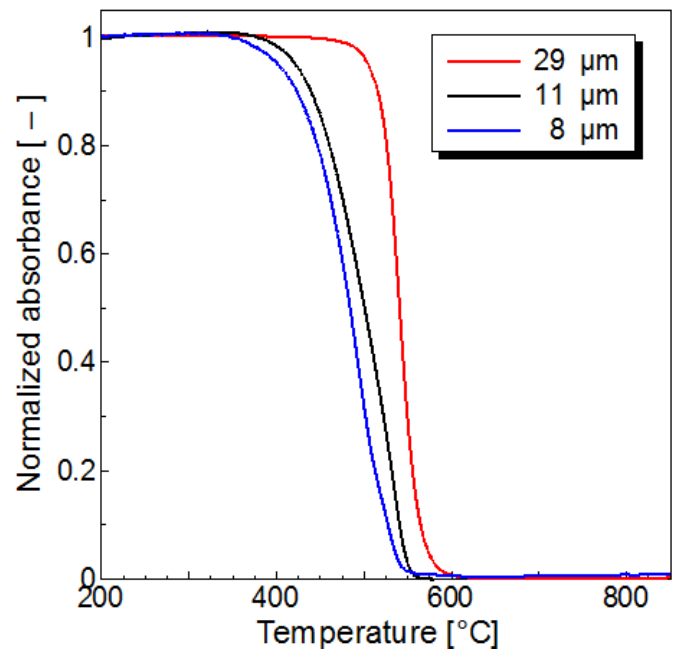

Fig. 10. Thermal degradation measurement obtained using the TOA method for VA-SWNT films synthesized under the same conditions but grown to different thickness. The thicker films show higher thermal stability. 